\title{
Determination of Ginseng with Different Ages Using a Taste-Sensing System
}

\author{
Shaoqing Cui, Jun Wang*, Lihua Geng ${ }^{1}$, Zhenbo Wei and Xiaojing Tian \\ Department of Biosystems Engineering, Zhejiang University, \\ 388 Yuhangtang Road, Hangzhou 310058, P. R. China \\ ${ }^{1}$ Ensoul Technology Ltd., \\ No. 168 Guanganmenwai Street, Xuanwu District, Beijing 100055, P. R. China
}

(Received July 23, 2012; accepted October 24, 2012)

Key words: taste-sensing system, artificial lipid-based membrane, ginseng, ages, ginsenoside content

In the medicinal herb market, adulteration of ginseng at different ages is commonly observed, which harms the health of consumers. Changes in the taste properties of ginseng at different ages were investigated using an intelligent taste-sensing system with artificial lipid-based membrane sensors and an ultraviolet spectrophotometer. It was found that the ginsenoside content increased linearly with increasing age, with the determination coefficient $\left(R^{2}\right)$ reaching 0.951. Results from the taste-sensing system provided rich information for the tested ginseng samples. The radar curve of taste value indicated that ginseng samples of different ages showed various taste characteristics and some certain tendencies. A linear correlation was established between ginseng age and the taste values of sourness, saltiness, and umami, with the determination coefficients $\left(R^{2}\right)$ being $0.941,0.943$, and 0.974 respectively. Taste data were analyzed using principal component analysis (PCA) and discriminant function analysis (DFA). A difference was obtained among groups with the first two PCs reaching 92.25\%. While in DFA a significant difference was observed with the first two function scores reaching 99.4\%. From the viewpoint of sensor response, the responses of the umami sensor AAE, saltiness sensor CTO, and sourness sensor CAO increased over the first $30 \mathrm{~s}$, while those of the bitterness sensor $\mathrm{COO}$ and astringency sensor AE1 decreased and remained at maximum in the negative value continuously, which provided additional information for the determination of ginseng samples at different ages. On the basis of integrated and special taste information, the ginseng growing year might be identified from the taste evaluation angles. A flow chart for further research on the taste features was suggested. This study intimated possible indexes for determining adulteration of ginseng at different ages by using an intelligent taste-sensing system.

${ }^{*}$ Corresponding author: e-mail: jwang@zju.edu.ch 


\section{Introduction}

Panax Ginseng, mostly cultivated in China and the Korean Peninsula, has been used as a kind of precious medicinal herb for thousands of years. Ginseng possesses immunestimulatory properties and strengthens the body's resistance, ${ }^{(1,2)}$ and has a wide range of therapeutic and pharmacological functions. ${ }^{(3)}$ However, with ginseng attracting more and more attention, fraudulence has become prevalent in the herb market due to ginseng's enormous economic potential, which leads to serious health damage to customers. The adulteration of ginseng at different ages is particularly common. Currently, two types of discrimination techniques have been applied to solve this problem. One is sensory evaluation; the other is measurement with sophisticated analysis instruments.

Traditional sensory evaluation (organoleptic test) has been frequently used in the herb market, but it is affected by the test conditions and panelists' personal preference. On the other hand, sophisticated analytical instruments, such as gas chromatography (GC), gas chromatography-mass spectrometry (GC-MS), and high-performance liquid chromatography (HPLC) are employed when physical and chemical indicators are required, ${ }^{(4-6)}$ but they are costly, time-consuming, and complicated. Both types of evaluation seem to be inaccurate, inconvenient, and time-consuming, which are opposite to the requirement of the determination methods used in the medicinal herb market. Therefore, it is necessary to develop cost-effective, convenient, and accurate technologies for the identification of adulterated ginseng at different ages.

Theaste-sensing system that mimics the human gustatory system might be used for the identification of adulterated ginseng at different ages. During the past decades, four models (SA401, SA402, SA402B, and TS-5000Z) of the taste-sensing system ${ }^{(7)}$ have been developed as an objective and precise sensing technology by Professor Toko and his group. ${ }^{(8-10)}$ Among the four models, TS-5000Z (Intelligent Sensor Technology Inc. Atsugi-shi, Japan) consists of a sensor unit and management server. A total of 8 sensors can be connected to the unit, providing initial (sourness, bitterness, astringency, umami, and richness) and aftertaste (bitterness, astringency, and umami). Each sensor is composed of an artificial lipid-based membrane and a $\mathrm{Ag} / \mathrm{AgCl}$ electrode. When detecting samples, the electrical potential difference is recorded and used as the intensity of each sensor. This system has been used for evaluating the taste of foodstuffs, such as coffee, ${ }^{(11)}$ green tea, ${ }^{(12)}$ and medicines ${ }^{(13,14)}$ as well as crude drugs. ${ }^{(15-18)}$

At present, the main evaluation indicator for ginsengs is age, and it has been reported that older ginsengs have higher contents of active components, such as ginsenoside and volatile oil.(19) Thus, the ginsenoside content has been considered to be the evaluation indicator from the detection methods and medical application standpoints. ${ }^{(20)}$ However, the elements of the ginsenoside are complex and still under investigation. The detection of ginsenoside content not only required complicated preprocessing but is also expensive and time-consuming. Take the traditional ginsenoside determination using HPLC for example. The samples are required to be extracted at first then purified as much as possible; special chromatographic columns and standard compounds are also required, the test conditions need to be optimized, and the whole experiment will take at least several days. The content of volatile oil also shows high correlation with age, ${ }^{(5)}$ but volatile oil represents the same drawbacks above. According to the traditional sensory evaluation, taste characteristics might be useful and convenient for determining 
adulteration, but sensory evaluation is not objective and limited by different panelists. Therefore, the taste characteristics of ginseng samples with different ages were chosen for study using the taste-sensing system that provides not only objective data but is also considered to be convenient and accurate. Besides, there are few reports about distinguishing different ginsengs from the viewpoints of taste evaluation using taste sensors.

In this study, taste data from the taste-sensing system was analyzed to find the difference and similarity among ginseng samples with different ages. It is reported that the features of ginseng differ with growing age. ${ }^{(20)}$ The ginsenoside was extracted and measured using a Soxhlet extractor and an ultraviolet spectrophotometer, respectively. These experiments were carried out with the aim of finding the corresponding relationship among taste quality data and ginseng age, and further exploring the possible indexes or determination method for discriminating the adulteration of ginseng at different ages.

\section{Materials and Methods}

\subsection{Materials and experiment procedure}

\subsubsection{Ginseng materials and reagents}

Ginseng samples were obtained from the same batch of fresh ginseng roots of four different ages (individual roots with estimated ages of 2, 3, 4, and 5 years), at the same planting bases in Fusong county of Jilin province in October 2010, because the time for harvesting fresh ginseng is usually from September to October. Jilin province is the largest ginseng-producing region in China. After the ginseng roots were taken to the laboratory, they were dried at a constant temperature $\left(45^{\circ} \mathrm{C}\right)$ for $6 \mathrm{~h}$ and sliced.

The taste-sensing system required special reference and washing solutions, which are similar to human saliva. The reference solution is $0.3 \mathrm{mM}$ tartaric acid in $30 \mathrm{mM}$ potassium chloride. The washing solution is $100 \mathrm{mM}$ hydrochloric acid in $30 \%$ ethanol aqueous solution for the negatively charged artificial lipid membrane sensor probe; an aqueous solution of $100 \mathrm{mM}$ potassium chloride and $10 \mathrm{mM}$ potassium in $30 \%$ ethanol was used for the positive one. All the reagents above are analytically pure and purchased from Sigma Aldrich, USA. The internal solution for the lipid membrane sensor (3.3 $\mathrm{M}$ potassium chloride in saturated silver chloride solution) was provided by Intelligent Sensor Technology, Inc. (Atsugi-shi, Japan).

Ether, methanol, butyl alcohol, concentrated sulfuric acid, ethanol, and vanillin were purchased from Guoyao Chemical Agents Company, China. The ginsenoside Re standard was purchased from the National Institutes for Food and Drug Control, China.

\subsubsection{Experiment procedure}

At the beginning, the ginsenoside content was determined to verify the usability of the tested ginseng samples with different growing years. Therefore, the Soxhlet extractor (SER148/6, VELP, Italy) and ultraviolet spectrophotometer (DR6000, HACA, USA) were used as the extractor and measurement instrument, respectively. Then, sensor data and taste data from the taste-sensing system TS-5000Z (Intelligent Sensor Technology, Inc. Japan) were defined to be the initial data for analyzing and determining 
the characteristics of ginseng samples with different ages. Ginseng samples of different ages (individual roots with estimated ages of 2, 3, 4, and 5 years) were measured using the procedure above.

After drying and slicing, the samples were milled and prepared for the measurement using the taste-sensing system and the measurement of the ginsenoside content. The details are found in $\S \S 2.3 .1$ and 2.3.2. All the measurements were carried out in successive weeks and the samples were stored in a vacuum dryer before testing.

\subsection{Taste-sensing system}

The taste of ginseng roots at different ages was determined using the taste-sensing system TS-5000Z, a type of intelligent sensor technology, which almost mimics the human gustatory system. It is reported that, in the surface of the human tongue, the lipid bilayer membrane is the main active tissue that maintains special potential. When food is in the mouth, the potential changes owing to electronic interaction or hydrophobic interaction; then, potential signals are sent to the brain and taste evaluation is carried out. Similarly, the taste-sensing system mimics this process, and the artificial lipidbased membrane plays the same role of the lipid bilayer membrane of the tongue. Up to eight sensors are provided to the system, representing gustatory stimuli such as sourness, sweetness, saltiness, astringency, umami, and three types of bitterness. Each sensor consists of a $\mathrm{Ag} / \mathrm{AgCl}$ electrode and an artificial lipid-based membrane made with different designed polymers (Table 1). The sensor unit is used to evaluate two types of taste: initial taste (sourness, saltiness, bitterness, astringency, and richness) and aftertaste (bitterness, astringency, and richness), which represent the taste of food in the mouth and after swallowing the food, respectively (Table 1). ${ }^{(9)}$

Table 1

Sensors of the taste-sensing system.

\begin{tabular}{|c|c|c|}
\hline Sensor name & Taste sensitivity & Application and artificial lipid \\
\hline $\mathrm{AAE}$ & Umami & $\begin{array}{l}\text { Foodstuffs such as beverage, flavoring agents etc. } \\
\text { Phosphoric acid di(2-ethylhexyl) ester }\end{array}$ \\
\hline CTO & Saltiness & $\begin{array}{l}\text { Foodstuffs such as flavoring agents etc. } \\
\text { Tetradodecylammonium bromide 1-hexadecanol }\end{array}$ \\
\hline $\mathrm{CAO}$ & Sourness & $\begin{array}{l}\text { Foodstuffs such as fruit juices. } \\
\text { Phosphoric acid di(2-ethylhexyl) ester; Oleic acid; } \\
\text { Trioctylmetylammomium chloride }\end{array}$ \\
\hline $\mathrm{COO}$ & $\begin{array}{l}\text { Bitterness for acidic bitter } \\
\text { materials }\end{array}$ & $\begin{array}{l}\text { Foodstuffs, medicine, and crude drugs, such as quinine etc. } \\
\text { Tetradodecylammonium bromide }\end{array}$ \\
\hline AE1 & Astringency & $\begin{array}{l}\text { Foodstuffs, medicine, crude drugs, such as toxic herbs etc. } \\
\text { Tetradodecylammonium bromide }\end{array}$ \\
\hline BTO & $\begin{array}{l}\text { Bitterness for bitter } \\
\text { hydrochloride salts }\end{array}$ & $\begin{array}{l}\text { Foodstuffs, medicine, crude drugs. } \\
\text { Phosphoric acid di- } n \text {-decyl ester }\end{array}$ \\
\hline ANO & $\begin{array}{l}\text { Bitterness for basic bitter } \\
\text { materials }\end{array}$ & $\begin{array}{l}\text { Foodstuffs, medicine, crude drugs. } \\
\text { Phosphoric acid di- } n \text {-decyl ester }\end{array}$ \\
\hline GLO & Sweetness & $\begin{array}{l}\text { Foodstuffs and medicine. } \\
\text { Tetradodecylammonium bromide, gallic acid }\end{array}$ \\
\hline
\end{tabular}




\subsection{Preparation for detection}

\subsubsection{Preparation for taste-sensing system measurement}

For four different ginseng sample groups, slices of each group were milled into powder and screened with a 40 mesh sieve. Ginseng powder of $60 \mathrm{~g}$ was weighed and divided into 5 sets. Each set of ginseng powder $(12.5 \mathrm{~g})$ was added to $100 \mathrm{ml}$ of boiling water at the solid-liquid ratio of $1: 8$ and then extracted by sonication for $30 \mathrm{~min}$ at $55^{\circ} \mathrm{C}$. After cooling to ambient temperature, the mixture solution was filtered with cotton first and then with filter papers. Five sets of ginseng filtrate were obtained as measureable sample. $35 \mathrm{ml}$ of the filtrate in each sample cup was used for the taste-sensing system.

\subsubsection{Preparation for determination of ginsenoside content}

After the ginseng samples were dried, milled, weighed (5 g), and wrapped with filterpapers, ginseng powder was extracted with ether in the Soxhlet extractor for $1 \mathrm{~h}$. Then, the extracted ginseng powder was added to $1 \mathrm{ml}$ of ultrapure water and $20 \mathrm{ml}$ of butyl alcohol and extracted by sonication for $30 \mathrm{~min}$. The supernatant was adopted from the centrifugal mixture solution. After four cycles, the butyl alcohol solution was combined and added with water (twice the volume of the butyl alcohol solution) for the extraction. The butyl alcohol solution was kept and dried using a rotary evaporator, dissolved with methanol, and diluted to $10 \mathrm{ml}$.

A ginsenoside Re standard solution (20 mg of each ginsenoside Re was dissolved in $10 \mathrm{ml}$ of methanol) and ginsenoside solution were prepared; $50 \mu \mathrm{l}$ each of the standard solution and ginsenoside solution were transferred to tubes with a stopper and dried. $0.5 \mathrm{ml}$ of $8 \%$ vanillin-ethanol and $5 \mathrm{ml}$ of $72 \%$ concentrated sulfuric acid were added to the tubes, shaken, and the mixed solution was heated in a $60^{\circ} \mathrm{C}$ water bath and then cooled in an ice-water bath for $10 \mathrm{~min}$. The mixed reagent without ginsenoside was used as a reference. Finally, both were determined at $544 \mathrm{~nm}$ using an ultraviolet spectrophotometer. The content was calculated using the equation:

$$
X(\%)=\frac{m_{1} \times \frac{A_{2}}{A_{1}}}{m_{2}} \times 100 \%,
$$

where $X$ is the content rate, $m_{1}$ is the ginsenoside Re standard weight, $m_{2}$ is the ginseng powder weight, and $A_{1}$ and $A_{2}$ are the absorbances of the ginsenoside Re standard solution and ginseng sample solution, respectively.

\subsection{Measurements and data analysis}

\subsubsection{Taste-sensing system measurement}

Figure 1 shows the measurement procedure of the taste-sensing system (TS-5000Z). When the sensor unit was dipped in the samples and reference solution, the electronic potential in the samples $(V \mathrm{~s})$ and potential in the reference solution $(V \mathrm{r})$ were recorded, which was regarded as the zero point. After sample solution measurement, the sensor unit was rinsed briefly in the reference solution; then, the potential $\left(V \mathrm{r}^{\prime}\right)$ was recorded when the reference solution was measured again. The difference $(V \mathrm{~s}-V \mathrm{r})$ between 


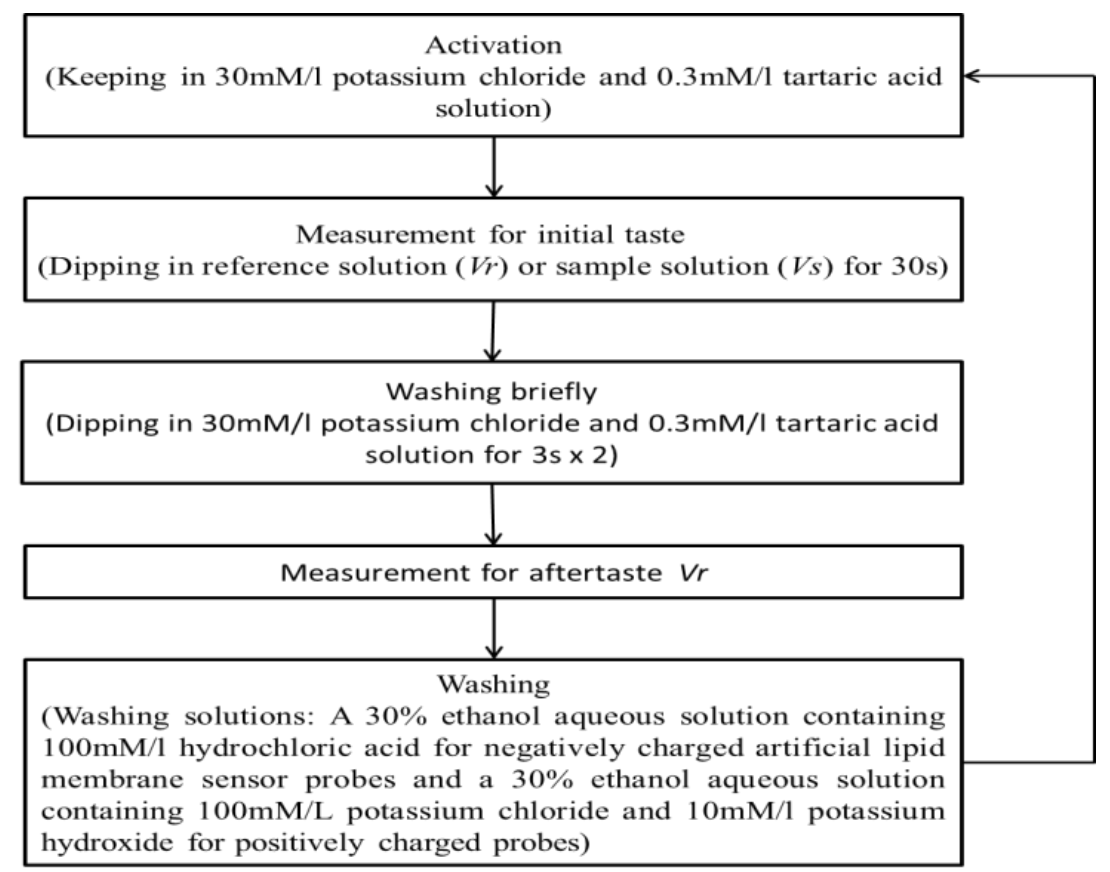

Fig. 1. Measurement procedures using the taste-sensing system TS-5000Z.

the sample potential $(V \mathrm{~s})$ and the reference potential $(V \mathrm{r})$ was the relative potential and used as the initial taste. The change in potential between before and after the reference solution $\left(V \mathrm{r}^{\prime}-V \mathrm{r}\right)$ was regarded as the aftertaste, which was regarded as the adsorption value (CPA). CPA is an abbreviation for change in membrane potential caused by adsorption. In this study, the testing of each group of ginseng samples was carried out following the procedures shown in Fig. 1.

\subsubsection{Data analysis}

Principal component analysis (PCA), discriminant function analysis (DFA), ${ }^{(21,22)}$ and regression analysis were employed to determine the significant difference between the studied ginseng samples of different ages. PLS ${ }^{(23)}$ was used to evaluate the correlation between the taste-sensing system data and growing years. PCA, DFA, and PLS analysis were carried out using SPSS; the figures were made with Origin and Microsoft Excel. 


\section{Results and Discussion}

\subsection{Relationship between ginsenoside content and growing years}

The change in ginsenoside content with increasing age has been studied by several researchers. It has been reported that the total contents of 12 ginsenosides in American ginseng roots at different ages increased gradually from 1-year-old to 5-year-old samples. ${ }^{(20)}$ More studies also demonstrated that the contents of ginseng components increase with the number of growing years. ${ }^{(24)}$ The correlation between ginsenoside content and age of ginseng was also investigated in order to inspect and verify the usability of ginseng materials, as shown in Fig. 2. The mean ginsenoside contents of these ginseng samples at 2, 3, 4, and 5 years were $0.98,1.83,2.50$, and $2.8 \%$, respectively, and the contents of both 4-year-old and 5-year-old samples were higher than or equal to $2.5 \%$. The Chinese Pharmacopoeia and the station standard use the ginsenoside content as an index to define the approximate ginseng age. It was reported that ginseng ages equal to or older than 4 years should contain at least $2.5 \%$ ginsenoside content. The inspected ginseng samples in this work fitted well with this definition. In Fig. 2, the total ginsenoside contents of ginseng roots show linear growth with increasing age, with the determination coefficient $R^{2}$ being 0.951 . The results inferred that ginseng samples with different ages had different characteristics, and some certain compositions changed regularly, such as ginsenoside. Moreover, it also showed that the chemical analysis method, which is the use of an ultraviolet spectrophotometer to determinine the ginsenoside content in this experiment, could be used to discriminate certain compositions from the viewpoint of detection technology. Although chemical analysis methods are applied to verify or determine substances, it is not difficult to find that they

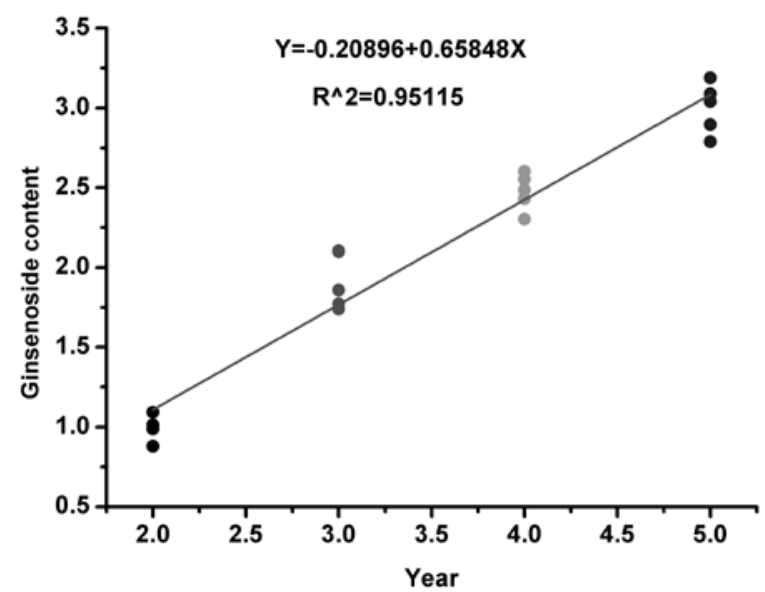

Fig. 2. Correlation between ginsenoside content and ginseng age. The five measured data of ginsenoside content for each type of ginseng sample were used to establish the relationship and regression equations. 
are mainly used to provide special results of certain substances rather than integrated information.

\subsection{Determination of ginseng samples with different ages using a taste-sensing} system

Compared with independent ginsenoside content results, the taste evaluations of ginseng samples from the taste-sensing system considered as promising comprehensive information were investigated in order to determine the adulteration of ginsengs with different ages. The information of taste values included not only initial taste (sourness, bitterness, astringency, umami, and richness) but also aftertaste (bitterness, astringency, and umami).

\subsubsection{Radar curve}

As shown in Fig. 3, the tested ginseng samples showed significant changes in multifarious taste values consisting of five initial tastes (astringency, bitterness, sourness, saltiness, and richness) as well as aftertastes of astringency and bitterness. Except for the taste of richness and aftertastes, the other five taste values of different aged ginseng samples showed different levels in Fig. 3. Among the initial tastes, the taste values of saltiness, sourness, and umami increased with the increase in ginseng age; meanwhile, the behaviors of bitterness and astringency changed sharply with increasing age. All the taste intensities were relative values and between the range from a negative value of -6 to a positive value of 4 , in comparison with the intensity of a 5-year-old ginseng sample, which was defined as zero. Therefore, negative values were observed. The bigger the

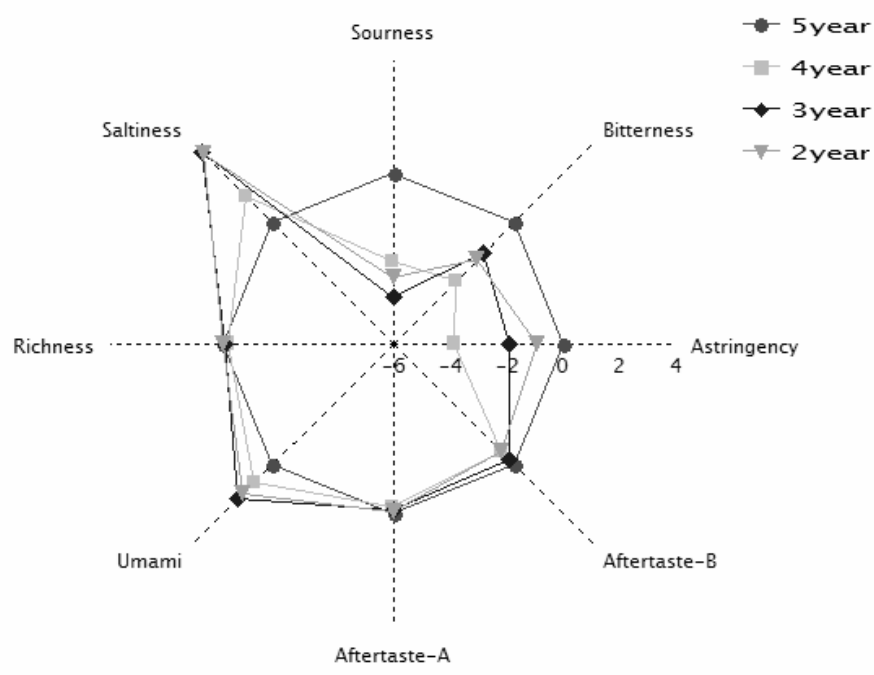

Fig. 3. Radar curves of taste data of ginseng samples at different ages. 
absolute values, the stronger the taste feeling. In detail, as for saltiness and umami, the ginseng samples with fewer growing years had higher intensity; in contrast, those with more growing years achieved lower intensity. As for sourness, with the increase in age, the taste level of sourness was enhanced. For bitterness and astringency, there was no strict rule between taste intensity and age; however, both taste levels decreased. In other words, these different ginseng samples with different ages showed different taste features as discussed above. Such taste level tendency mentioned above might be attributed to the active components in the ginseng samples. Considering the ginseng components, there might be some taste compounds or similar molecular structures, such as monosodium glutamate (umami), potassium chloride (saltiness), tartaric acid (astringency), tannic acid (sourness), and quinine hydrochloride (basic bitterness), which are standard materials for the taste-sensing system TS-5000Z (Intelligent Sensor Technology Inc. Atsugi-shi, Japan) for calculating, converting, and analyzing the taste level. ${ }^{(7)}$ Furthermore, the ginseng taste level evaluation from the experiment data agreed well with the description of the ginseng features in the Chinese Pharmacopoeia. The results may indicate that ginseng samples with different growing years presented different taste fingerprint information, and they might be used as indexes for the determination of adulteration of different ginseng samples.

\subsubsection{Relationship between taste values and ginseng growing years}

From the taste characteristics of ginseng samples mentioned above, it might be implied that the taste values of saltiness, sourness, and umami increased with increasing ginseng age. For the further study, it is necessary to know the correlation between the taste values and the ginseng ages. As show in Fig. 4(b), the taste values of saltiness, sourness, and umami changed linearly with increasing ginseng age from 3 to 5 years, although the taste values showed a curved correlation with the ginseng age from 2 to 5 years, as shown in Fig. 4(a). However, it is worth noting that 2-year-old ginseng is not used for adulterating in the herb market but mainly used for cultivation. The reason is that the price of the 2-year-old ginseng is almost the same as that of the 3-yearold ginseng, but the former is clearly smaller than the latter. Besides, the content of active components, such as ginsenoside content, is too low to be chosen for medicinal application. Actually, 2-year-old ginsengs are not easy to find in the market. Therefore, from the practical application viewpoint, the taste data of 2-year-old ginseng were not included in this correlation analysis. Figure 4(b) shows the correlation between saltiness, sourness, umami values and ginseng ages excluding the 2-year-old sample. It was found that these three taste values changed linearly with increasing age. In detail, the sourness values increased with increasing age with a determination coefficient $\left(R^{2}\right)$ of 0.974 , but the saltiness and umami values decreased with $R^{2}$ being 0.941 and 0.943 , respectively. These linear correlations are supposed to be applied for predicting ginseng ages in a certain range based on the high linear relationship. Lee et al. has also showed that chloroform, ethyl acetate, and $n$-butanol, which are sub-fractions from ginseng (P. ginseng), have a high affinity for binding with sourness, bitterness, and umami. ${ }^{(25)}$ The increased sourness could be interpreted by the change in the ethyl acetate (EtOAC) concentration, while umami and astringency are related to the $n$-butanol and 


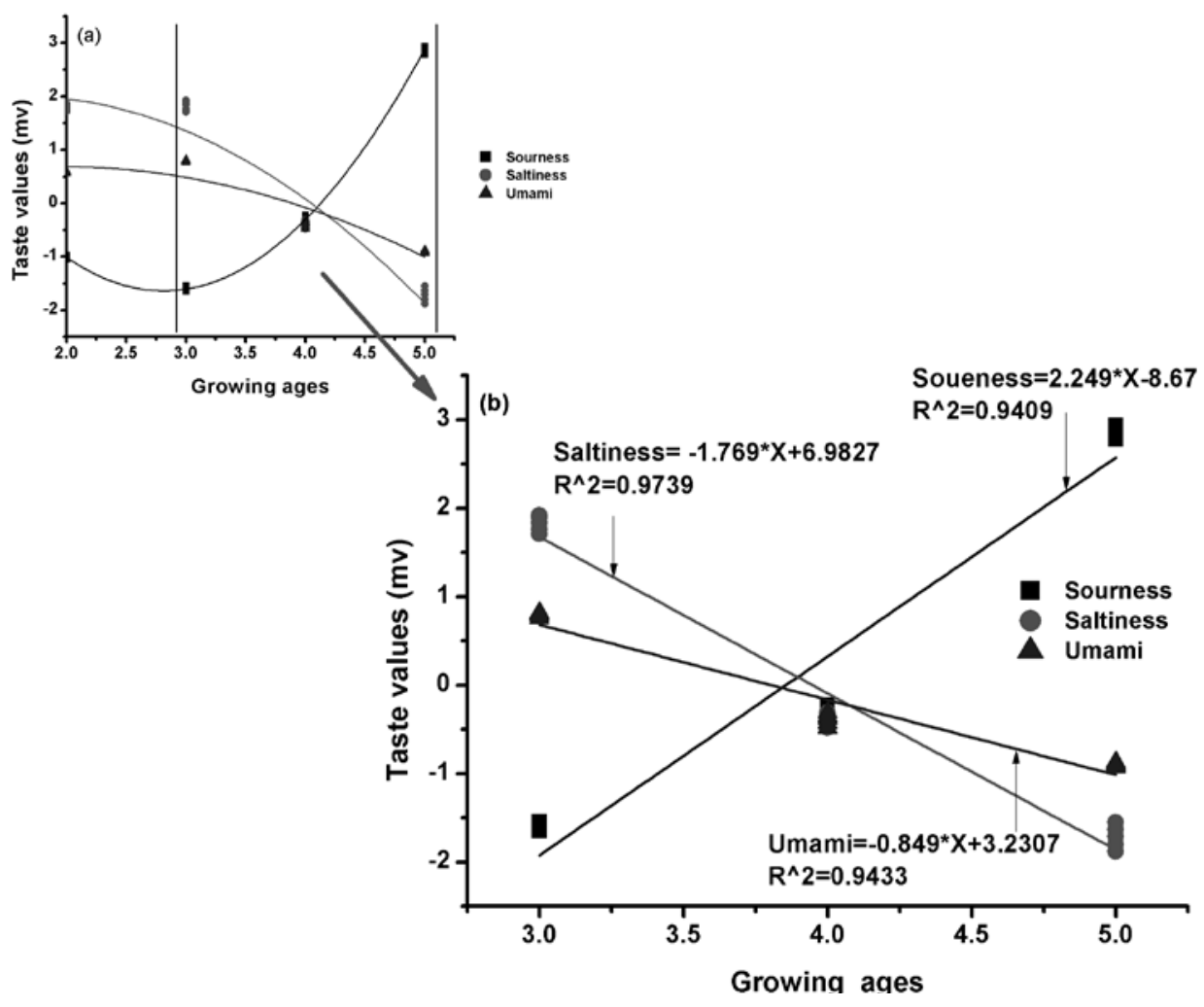

Fig. 4. Relationship between the three taste values (sourness, saltiness, and umami) and growing years of ginseng samples. Plot (a) is the correlation of tatse values and ginseng ages from 2 to 5 years, and plot (b) is the linear correlation of taste values and ginseng ages from 3 to 5 years.

polyphenols of ginseng extracts. ${ }^{(26)}$ In other words, the contents of some compositions, which probably accounted for some taste evaluations of sourness, umami, and saltiness in ginseng roots, increased or decreased linearly with the growing age. These taste evaluations and relation curves could become options for distinguishing different ginsengs with different ages. Moreover, it might be inferred that the taste-sensing system could indicate the quantitative change in chemical compositions during ginseng cultivation.

\subsubsection{PCA and DFA}

PCA and DFA based on taste data are shown in Fig. 5. Obviously, groups of 5, 4, 3, and 2 years were scattered in both plots with the first two principal components reaching $92.25 \%$ and the first two function scores reaching 99.4\%. Compared with the PCA 

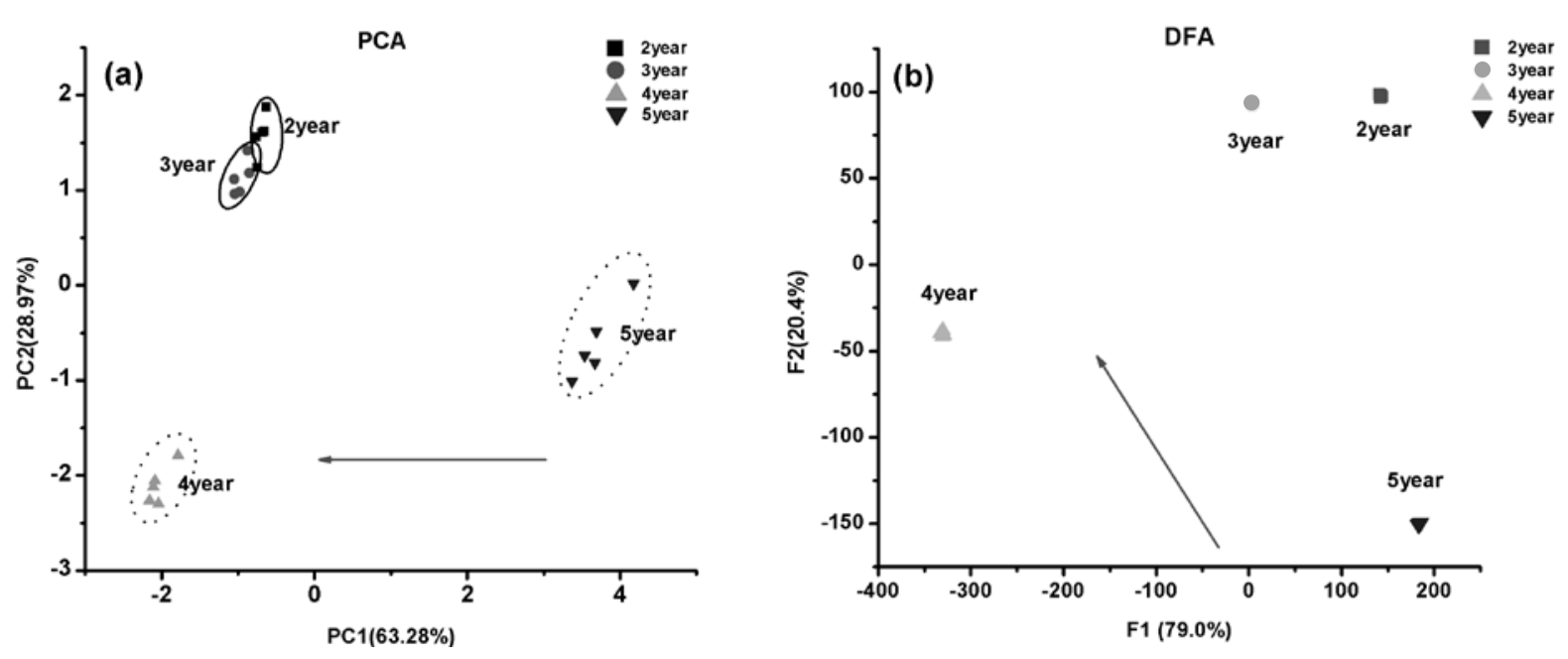

Fig. 5. PCA plot (a) and DFA plot (b) of ginseng samples with different growing years based on taste data after interpolation difference algorithm processing. A total of 5 experiment results of the taste-sensing system were collected in both analyses.

results, DFA performed better than PCA with all the ginseng groups dispersed markedly. In the PCA plot (Fig. 5(a)), the 3-year-old and 2-year-old ginseng groups were close to each other but far from both the 4-year-old and 5-year-old ginseng groups; at the same time, the two higher-aged groups were clearly separated from each other. Comparing both plots, it is not difficult to find that the distributions of different groups showed a certain tendency as the red arrows on the maps in both plots. In detail, the number of growing years decreased along with direction of the arrow. In other words, it indicated that the taste of ginseng samples with different growing ages changed following a certain degree of regularity. Although groups of 2- and 3-year-old ginseng samples were next to each other in the PCA plot (Fig. 5(a)), they separated distinctly in the DFA plot. This might be interpreted using the principle of DFA algorithm, which is based on the known classification number. However, it suggested that the difference was observed using DFA between 2- and 3-year-old ginseng samples based on the taste-sensing data. Moreover, this result also indicated that the slight difference between the two types of ginseng samples of lower ages could be distinguished clearly by the taste-sensing system from the application of discrimination standpoints.

\subsubsection{Sensor response}

It is well known that the sensor unit attached with artificial lipid membranes is the most important part of the taste-sensing system, which plays a role as the taste buds concentrated on the tongue surface. In this study, this sensor unit consisting of five sensors (AAE, CTO, CAO, COO, and AE1) showed the change and tendency of the 
electronic potential directly once they detected ginseng aqueous solutions. As shown in Fig. 6, the ginseng aqueous solution had significant effects on taste sensors (artificial lipid membrane). The responses of the umami sensor AAE, saltiness sensor CTO, and sourness sensor CAO increased over $30 \mathrm{~s}$, but those of the bitterness sensor $\mathrm{COO}$ and astringency sensor AE1 showed contrary behaviors. The sensor AE1 response decreased sharply in the first $10 \mathrm{~s}$, and it reached and remained at the negative maximum value of 2 from 10 to $30 \mathrm{~s}$. The sensor COO response reduced slowly over $30 \mathrm{~s}$. The behaviors of sensors $\mathrm{COO}$ and $\mathrm{AE} 1$ indicated that bitterness and astringency tastes might be first impression tastes, because they almost reached the negative maximum values in the first $10 \mathrm{~s}$. Then, the sourness and saltiness tastes were detected, making the taste become more unpleasant because the response of sensors CAO and CTO kept increasing over 30 $\mathrm{s}$ and almost reached a value of 2 at $15 \mathrm{~s}$. In fact, it was reported that the taste of ginseng is bitter and astringent, ${ }^{(27)}$ which fits with the description of the above sensor behaviors. That means that the sensor response behaviors might describe the taste changes when people imbibe ginseng aqueous solution. In reference to the quantitative analysis discussed above, the sensor response might be regarded as a qualitative analysis for the reason of showing the first impression or the most direct evaluation for different ginseng samples, which could be compared with the response of the human tongue.

\subsection{A flow chart for further investigation}

Admittedly, it is possible to discriminate the taste characteristics of different types of ginseng samples with different ages by using the taste-sensing system, while more

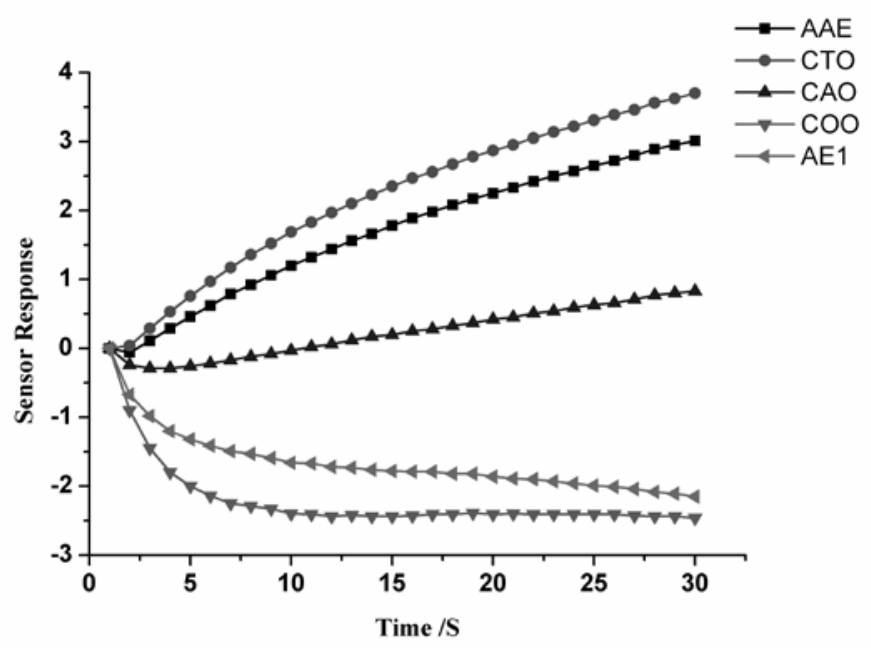

Fig. 6. One of the sensor responses of 5-year-old ginseng over the first $30 \mathrm{~s}$. The values of the vertical axis are relative electric potential calculated with $\left(V_{0}-V\right) / V_{0}$, where $V_{0}$ is the potential at $t=$ 0 (baseline resistance) and $V$ is the potential at the selected time. 
ginseng samples should be tested to complete the taste characteristics or results discussed above. Furthermore, a database based on plenty of taste information might be required, which records the taste features of different ginseng samples, to meet the needs of the herb market. This process as shown in Fig. 7 might seem like labeling management. In the chart, more known ginseng samples, whose taste data are required to be tested and measured continually from seedling stages to different harvest times, are used to provide precise data and perfect the database. Then, taste data would be discussed and analyzed as mentioned in this paper. If possible, the relationship between taste data and ginseng age might be established based on the database to predict ages or determine the adulteration of different ages for ginsengs.

From the results and discussion, it is not difficult to find that the taste-sensing system provides more information than chemical analysis methods, and the sensors provide more reliable data. That is because the taste data is more objective than sensory evaluation, and the results were analyzed by synthetic judgment based on the integrated data. On the other hand, the sensor response might show the changes happening in the mouth when foodstuffs are taken and might be considered additional information for discriminating samples.

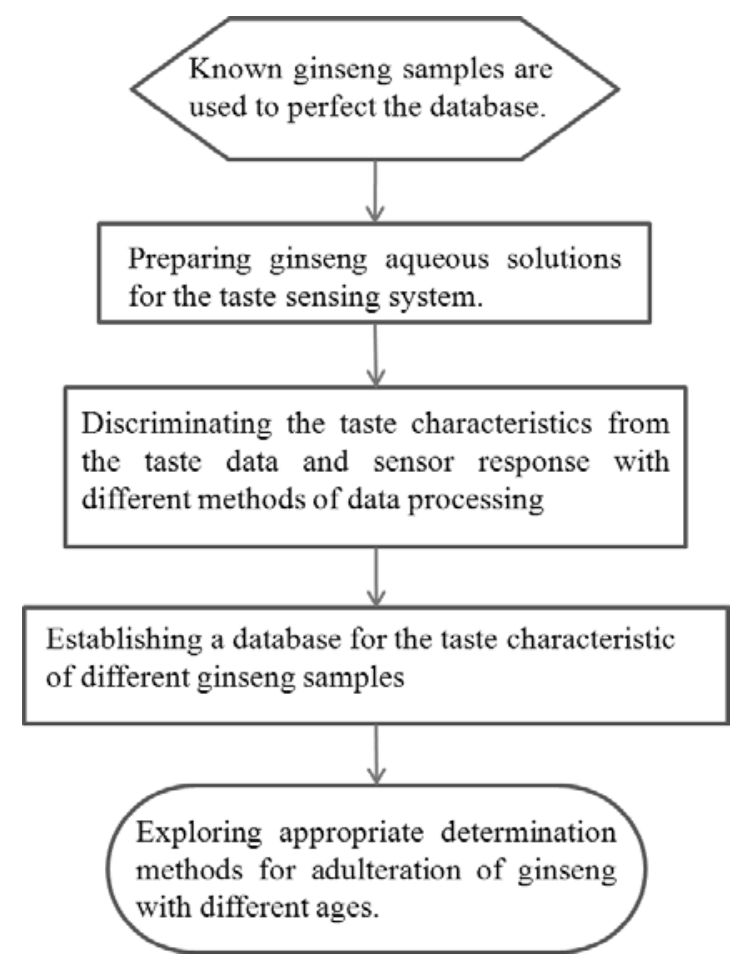

Fig. 7. A flow chart for further investigation. 


\section{Conclusions}

The determination of ginseng at different ages was explored with the taste-sensing system and an ultraviolet spectrophotometer. Taste data from the taste-sensing system seemed to be richer than traditional physiochemical index. In this study, at the beginning, a correlation was established between ginsenoside content and growing ages with the correlation coefficient $\left(R^{2}\right)$ being 0.951 . According to the results obtained from the taste-sensing system, different characteristics and tendencies were observed from the radar curves, correlation between the taste values and ginseng ages, PCA and DFA discrimination as well as sensor response. Radar curves showed that the taste values of saltiness, sourness, and umami increased with ginseng age. Relationships were established and compared between three tastes (sourness, saltiness, and umami) and ginseng ages with $R^{2}$ reaching 0.974 (saltiness), 0.941 (sourness), and 0.943 (umami). DFA provided better performance than PCA with the first two function scores reaching 99.4\%, while the first two PCs were $92.25 \%$. The sensor responses of AAE, CTO, CAO, $\mathrm{COO}$, and AEE changed gradually and significantly over the first $30 \mathrm{~s}$, indicating that the five sensors showed high sensitivity to the tested ginseng samples. Moreover, the behaviors of the sensor response possibly provided additional taste features once the sensors were emersed in the aqueous solutions. Compared with the chemical analysis, the taste-sensing system provided relatively integrated and special taste characteristics rather than single physicochemical index information.

\section{Acknowledgements}

The experiments were supported by Ensoul Technology Ltd. The authors gratefully acknowledge the technical guidance from Engr. Guisheng Chang and Engr. Yang Li, as well as writing guidance from Dr. Ronggang Chen of Intelligent Sensor Technology, Inc.

\section{References}

1 S. K. Lee, S. H. So, E. Hwang, B. S. Koo, G. H. Han and N. Kim: J. Korean Soc. Food Sci. Nutr. 37 (2008) 434.

2 H. R. Kim, Y. Cui, S. J. Hong, S. J. Shin, D. S. Kim, N. M. Kim, S. H. So, S. K. Lee, E. C. Kim, S. W. Chae and H. J. Chea: Immunopharmacol. Immunotoxicol. 30 (2008) 333.

3 S. S. Joo, T. J. Won and D. I. Lee: Planta Med. 71 (2005) 476.

4 S. K. Lee, J. H. Kim, H. J. Sohn and J. W. Yang: Sens. Actuators, B 106 (2005) 7.

5 Y. Q. Qiu, X. Lu, T. Pang, C. F. Ma, X. Li and G. W. Xu: J. Sep. Sci. 31 (2008) 3451.

6 M. R. Kim, Y. Lee, B. J. Park, J. H. Choi, I. S. Kim and J. H. Shim: Korean J. Pestic. Sci. 9 (2005) 237.

7 Y. Kobayshi, M. Habara, H. Ikezazki, R. Chen, Y. Naito and K. Toko: Sensors 10 (2010) 3411 .

8 K. Toko: Biomimetic Sensor Technology (Cambridge University Press, Cambridge, 2000) p. 113.

9 K. Toko: Meas. Sci. Technol. 9 (1998) 1919.

10 K. Toko: Sens. Actuators, B 64 (2000) 205.

11 T. Fukunaga, K. Toko and K. Mori: Sens. Mater. 8 (1996) 47. 
12 N. Hayashi, R. Chen, H. Ikezaki, S. Yamaguchi, D. Maruyama, Y. Yamaguchi, T. Ujihara and K. Kohata: Biosci. Biotechnol. Biochem. 70 (2006) 626.

13 K. Woertz, C. Tissen, P. Kleinebudde and J. Breitkreutz: Int. J. Pharm. 417 (2011) 256.

14 K. Woertz, C. Tissen, P. Kleinebudde and J. Breitkreutz: J. Pharm. Biomed. Anal. 55 (2011) 272 .

15 N. Anjiki, N. Kawahara and Y. Goda: Nat. Med. 59 (2005) 64.

16 N. Anjiki, A. Suzuki, N. Kawahara and Y. Goda: Jpn. J. Pharmacogn. 60 (2006) 21.

17 N. Anjiki, C. Yoshino, N. Kawahara and Y. Goda: Jpn. J. Pharmacogn. 61 (2007) 6.

18 N. Anjiki, J. Hosoe, H. Fuchino, F. Kiuchi, S. Sekita, H. Ikezaki, M. Mikage, N. Kawahara and Y. Goda: J. Nat. Med. 65 (2011) 293.

19 J. Wang, L. X. Zhang and Y. Zhao: Med. Plant 2 (2011) 40.

20 C. L. Qu, Y. P. Bai, X. Q. Jin, Y. T. Wang, K. Zhang, J. Y. You and H. Q. Zhang: Food Chem. 115 (2009) 340.

21 H. C. Yu, J. Wang, C. Yao, H. M. Zhang and Y. Yu: LWT Food Sci. Technol. 41 (2008) 1268.

22 H. Yu, J. Wang, H. Zhang, Y. Yu and C. Yao: Sens. Actuators, B 128 (2008) 455

23 H. M. Zhang, M. X. Chang, J. Wang and S. Ye: Sens. Actuators, B 134 (2008) 332.

24 I. M. Chung, J.-W. Kim, P. Seguin, Y.-M. Jun, S.-H. Kim: Food Chem. 130 (2012) 73

25 D. G. Lee, K.-T. Kim and S. H. Lee: Afr. J. Biotechnol. 11 (2012) 9280.

26 A. E. Hagerman and L. G. Bulter: J. Biol. Chem. 256 (1981) 4494.

27 S. Y. Hu: Econ. Bot. 30 (1976) 11. 